\title{
Subspace formulation of time-dependent density functional theory for large-scale calculations
}

\author{
Xu Zhang and Gang Lu \\ Department of Physics and Astronomy, California State University Northridge, \\ Northridge, California 91330-8268, USA
}

(Received 31 March 2015; accepted 31 July 2015; published online 14 August 2015)

\begin{abstract}
A subspace formulation of time-dependent density functional theory (TDDFT) is proposed for largescale calculations based on density functional perturbation theory. The formulation is implemented in conjunction with projector augmented-wave method and plane-wave basis set. A key bottleneck of conventional TDDFT method is circumvented by projecting the time-dependent Kohn-Sham eigenvalue equations from a full Hilbert space to a substantially reduced sub-Hilbert space. As a result, both excitation energies and ionic forces can be calculated accurately within the reduced subspace. The method is validated for several model systems and exhibits the similar accuracy as the conventional TDDFT method but at a computational cost of the ground state calculation. The Born-Oppenheimer molecular dynamics can be successfully performed for excited states in $\mathrm{C}_{60}$ and $\mathrm{T}_{12}$ molecules, opening doors for many applications involving excited state dynamics. (C) 2015 AIP Publishing LLC. [http://dx.doi.org/10.1063/1.4928510]
\end{abstract}

\section{INTRODUCTION}

Understanding excited state phenomena is at the heart of many important materials problems, such as photovoltaics, photocatalysis, plasmonics, solid-state lighting, and biochemical sensing, to name a few. Within the realm of firstprinciples simulations, time-dependent density functional theory (TDDFT) $)^{1,2}$ has become one of the most powerful, versatile, and popular tools for probing electronic structure and excitations in molecular and solid state materials; ${ }^{3-7}$ TDDFT has been programmed in most quantum chemistry and quantum physics electronic structure codes and is the most widely used method to treat excited states in medium- to large-sized molecules. ${ }^{8,9}$ However, despite the tremendous success of TDDFT, its application to practical problems remains challenging, in a large part owing to its high computational demand.

For a large system with more than a few hundred atoms, three intertwined computational bottlenecks are often encountered in TDDFT calculations. The first bottleneck is to self-consistently determine the ground state charge density and Kohn-Sham (KS) Hamiltonian based on the occupied KS eigenstates whose number is $N_{\text {occ. }}$ The second bottleneck is to diagonalize the KS Hamiltonian and obtain the full spectrum of $\mathrm{KS}$ eigenstates, whose dimension is $N_{\text {occ }}+N_{\text {virt }}$ with $N_{\text {virt }}$ being the number of virtual KS orbitals. The third bottleneck is to solve time-dependent Kohn-Sham (TDKS) eigenvalue equations with the matrix dimension of $N_{\text {occ }} \times N_{\text {virt }}$. To overcome the first bottleneck, one often resorts to plane-wave pseudopotential methods thanks to simplicity, completeness, and the Fast Fourier Transform (FFT) algorithm afforded by the plane-wave basis. Another advantage of the plane-wave pseudopotential methods is that they are amenable to extended condensed matter systems. To address the two other bottlenecks, one has to reduce the dimension of full KS spectrum and the dimension of TDKS matrix. To this end, Doltsinis and Kosov ${ }^{10}$ proposed a planewave TDDFT method that used only a subset of KS virtual orbitals to construct the TDKS eigenvalue equations. However, although the excitation energies are usually acceptable, ${ }^{11-14}$ the ionic forces converge much slower with respect to the dimension of the subset, resulting in substantial errors. On the other hand, based on the density functional perturbation theory (DFPT), ${ }^{15,16}$ Hutter $^{17}$ recast the plane-wave TDDFT formulation to eliminate explicit references to the virtual KS orbitals, thus reducing the dimension of the KS spectrum. However, since the third bottleneck stands unresolved, the computational cost of Hutter's method remains high for a large system.

Here, we propose a plane-wave TDDFT method based on the density functional perturbation theory, which renders a clean separation of Hilbert spaces for the solution of TDKS eigenvalue equations. In particular, one can project the TDKS equations from a full Hilbert space to a substantially reduced sub-Hilbert space, thus circumventing the third bottleneck. Although there are TDDFT methods which can obtain accurate excitation energies, the proposed approach is the only one to the best of our knowledge - that can determine both energy and ionic force of excited states accurately for a large system. By simultaneously removing the three bottlenecks, the proposed method can significantly reduce the computational cost of large-scale TDDFT calculations and thus paves ways to many applications that are presently beyond the reach of TDDFT. The method is general and can be implemented in conjunction with other basis functions and/or pseudopotentials, such as real space grids, ${ }^{18,19}$ ultrasoft pseudopotential, ${ }^{20}$ and projector augmented-wave (PAW). ${ }^{21,22}$ In this paper, we focus on the implementation of the method within the planewave PAW framework. 
This paper is organized as follows. We first present the theoretical formulation of the proposed subspace TDDFT method. We then examine its accuracy and efficiency by applying the method to various benchmark systems, including $\mathrm{CH}_{2} \mathrm{O}, \mathrm{N}_{2}, \mathrm{C}_{60}$, and $\mathrm{T}_{12}$ molecules as well as disordered poly(3-hexylthiophene) (P3HT) conjugated polymers. A computational scaling analysis of the method and brief discussion on why the method would work are provided. Finally, we summarize the work and envision future development of the method.

\section{METHODOLOGY}

In the following, we use indices $i, j, k, \ldots$ to label occupied KS orbitals and $\sigma, \tau$ to denote their spins. In the PAW method, the all-electron (AE) wave-function $\left|\psi_{i \sigma}\right\rangle$ is a linear transformation of the pseudo-wave-function (PS) $\left|\widetilde{\psi}_{i \sigma}\right\rangle$, i.e., $\left|\psi_{i \sigma}\right\rangle=\mathcal{T}\left|\widetilde{\psi}_{i \sigma}\right\rangle$, and the KS equation is expressed as $\widetilde{H}^{\sigma}\left|\widetilde{\psi}_{i \sigma}\right\rangle=\sum_{j} \epsilon_{i j \sigma} \widetilde{S}\left|\widetilde{\psi}_{j \sigma}\right\rangle$ with the orthonormal condition $\left\langle\widetilde{\psi}_{i \sigma}|\widetilde{S}| \widetilde{\psi}_{j \tau}\right\rangle=\delta_{i j} \delta_{\sigma \tau}$, where $\widetilde{S}$ is overlap operator. A brief description of the PAW formalism is presented in Appendix A, and more details can be found elsewhere. ${ }^{21,22}$ In the presence of a harmonic perturbation potential with a frequency $\omega$, the linear response of the electron charge density is given by

$$
\Delta \widetilde{\rho}^{\sigma}( \pm \omega)=\sum_{i}\left(\left|\widetilde{\psi}_{i \sigma}\right\rangle\left\langle\widetilde{\psi}_{i \sigma}^{\mp}|+| \widetilde{\psi}_{i \sigma}^{ \pm}\right\rangle\left\langle\widetilde{\psi}_{i \sigma}\right|\right)
$$

in the frequency domain. Here, $\left|\widetilde{\psi}_{i \sigma}^{ \pm}\right\rangle$are the linear response orbitals which are chosen to be orthogonal to the occupied orbitals, i.e., $\left\langle\widetilde{\psi}_{i \sigma}^{ \pm}|\widetilde{S}| \widetilde{\psi}_{j \sigma}\right\rangle=0$ based on the DFPT formulation. ${ }^{15}$ The excitation energies $\omega$ are given by the solutions to the equation

$$
\sum_{j}\left(\widetilde{H}^{\sigma} \delta_{i j}-\epsilon_{i j \sigma} \widetilde{S}\right)\left|\widetilde{\psi}_{j \sigma}^{ \pm}\right\rangle+\widetilde{P}_{\mathrm{c}}^{\sigma} \Delta \widetilde{V}_{\mathrm{eff}}^{\sigma}( \pm \omega)\left|\widetilde{\psi}_{i \sigma}\right\rangle=\mp \omega \widetilde{S}\left|\widetilde{\psi}_{i \sigma}^{ \pm}\right\rangle
$$

where $\widetilde{P}_{\mathrm{c}}^{\sigma}=1-\sum_{i} \tilde{S}\left|\widetilde{\psi}_{i \sigma}\right\rangle\left\langle\widetilde{\psi}_{i \sigma}\right|$ is the projection operator onto the space, $\Omega^{\text {virt }}$ spanned by the virtual KS orbitals. The perturbed KS effective potential is given by

$$
\Delta \widetilde{V}_{\mathrm{eff}}^{\sigma}( \pm \omega)=\sum_{\tau} \frac{\delta \widetilde{V}_{\mathrm{eff}}^{\sigma}}{\delta \widetilde{\rho}^{\tau}} \Delta \widetilde{\rho}^{\tau}( \pm \omega) \equiv \sum_{\tau} \widetilde{K}_{\sigma \tau} \Delta \widetilde{\rho}^{\tau}( \pm \omega) .
$$

Here, $\widetilde{K}_{\sigma \tau}$ is the kernel functional whose detailed expression can be found in Appendix B.

In the following, we only consider the spin-restricted case on the $\Gamma$-point in the Brillouin zone, where the KS orbitals are taken to be real. Introducing unitary transformations,

$$
\widetilde{u}_{i}^{ \pm \pm}=\frac{1}{\sqrt{2}}\left(\left|\widetilde{\psi}_{i \uparrow}^{ \pm}\right\rangle+\left|\widetilde{\psi}_{i \downarrow}^{ \pm}\right\rangle\right), \widetilde{u}_{i}^{\mathrm{T}}=\frac{1}{\sqrt{2}}\left(\left|\widetilde{\psi}_{i \uparrow}^{ \pm}\right\rangle-\left|\widetilde{\psi}_{i \downarrow}^{ \pm}\right\rangle\right),
$$

we can derive a non-Hermitian eigenvalue equation

$$
\left(\begin{array}{cc}
\widetilde{\mathbf{A}} & \widetilde{\mathbf{B}} \\
\widetilde{\mathbf{B}} & \widetilde{\mathbf{A}}
\end{array}\right)\left(\begin{array}{l}
\widetilde{\mathbf{u}}^{+} \\
\widetilde{\mathbf{u}}^{-}
\end{array}\right)=\omega\left(\begin{array}{cc}
-\widetilde{\mathbf{S}} & 0 \\
0 & \widetilde{\mathbf{S}}
\end{array}\right)\left(\begin{array}{c}
\widetilde{\mathbf{u}}^{+} \\
\widetilde{\mathbf{u}}^{-}
\end{array}\right),
$$

which is analogous to Casida's equation ${ }^{3}$ for both singlet ("S") and triplet ("T") excited states. The operators $\widetilde{\mathbf{A}}$ and $\widetilde{\mathbf{B}}$ act on the orbitals according to

$$
\begin{gathered}
{[\widetilde{\mathbf{A}} \widetilde{\mathbf{u}}]_{i}=\sum_{j}\left(\widetilde{H} \delta_{i j}-\epsilon_{i j} \widetilde{S}\right)\left|\widetilde{u}_{j}\right\rangle+\sum_{j}\left\langle\widetilde{P}_{\mathrm{c}} \widetilde{\psi}_{i}|\widetilde{K}| \widetilde{\psi}_{j} \widetilde{P}_{\mathrm{c}} \widetilde{u}_{j}\right\rangle,} \\
{[\widetilde{\mathbf{B}} \widetilde{\mathbf{u}}]_{i}=\sum_{j}\left\langle\widetilde{P}_{\mathrm{c}} \widetilde{\psi}_{i}|\widetilde{K}| \widetilde{P}_{\mathrm{c}} \widetilde{u}_{j} \widetilde{\psi}_{j}\right\rangle .}
\end{gathered}
$$

Since $\widetilde{u}_{i}$ belongs to $\Omega^{\text {virt }}$, it should also belong to the product space $\Omega_{i}^{\text {virt }}=\Omega^{\text {virt }} \otimes \Omega_{i}$; here, $\Omega_{i}$ represents one dimensional space spanned by the $i$ th occupied KS orbital. Eq. (3) is then iteratively solved ${ }^{23}$ in the full Hilbert space $\Omega_{1}^{\text {virt }} \oplus \Omega_{2}^{\text {virt } \ldots}$ $\oplus \Omega_{N_{\text {occ }}}^{\text {virt }}$ to determine the low-lying excited states. As alluded to earlier, when $N_{\text {occ }}$ is large, the solution of Eq. (3) becomes very expensive. Fortunately, in many systems, only a subset of occupied KS orbitals actually responds to the perturbation potential, i.e., $\widetilde{\psi}_{i \sigma}^{ \pm}=0$ for $i \notin N_{\mathrm{v}}$ where $N_{\mathrm{v}}$ represents the size of the subset. Therefore, we propose to solve Eq. (3) in a sub-Hilbert space $\Omega_{1}^{\text {virt }} \oplus \Omega_{2}^{\text {virt }} \cdots \oplus \Omega_{N_{v}}^{\text {virt }}$ as opposed to the full Hilbert space. For example, the singlet excited state ${ }^{1} \Sigma_{u}^{-}$of a $\mathrm{N}_{2}$ molecule involves excitations from occupied $\pi$ orbitals only, not $\sigma$ orbitals. In addition, for a low-lying excitation in a large system, the contributions from deeply occupied orbitals are often negligible.

Next we introduce a Lagrangian functional $\mathcal{L}_{\text {ex }}$ whose derivatives are discussed below. Most importantly, its derivative with respect to ionic position $R$ gives rise to the ionic force,

$$
\begin{aligned}
\mathcal{L}_{\text {ex }}\left[\widetilde{\mathbf{u}}^{ \pm}, \omega, \widetilde{\mathbf{Z}}, \boldsymbol{\Lambda}, \boldsymbol{\Gamma}, \widetilde{\psi}\right] & \\
= & \mathcal{L}\left[\widetilde{\mathbf{u}}^{ \pm}, \omega\right]+\sum_{i}\left\langle\widetilde { Z } _ { i } \left|\left(\widetilde{H}\left|\widetilde{\psi}_{i}\right\rangle-\sum_{j} \Lambda_{i j} \widetilde{S}\left|\widetilde{\psi}_{j}\right\rangle\right)\right.\right. \\
& -\sum_{i \geq j} \Gamma_{i j}\left(\left\langle\widetilde{\psi}_{i}|\widetilde{S}| \widetilde{\psi}_{j}\right\rangle-\delta_{i j}\right),
\end{aligned}
$$

which is similarly defined in previous works. ${ }^{17,24,25}$ The first term is given by

$$
\begin{aligned}
\mathcal{L}\left[\widetilde{\mathbf{u}}^{ \pm}, \omega\right]= & \frac{1}{2}\left(\widetilde{\mathbf{u}}^{+}+\widetilde{\mathbf{u}}^{-}\right)(\widetilde{\mathbf{A}}+\widetilde{\mathbf{B}})\left(\widetilde{\mathbf{u}}^{+}+\widetilde{\mathbf{u}}^{-}\right) \\
& +\frac{1}{2}\left(\widetilde{\mathbf{u}}^{+}-\widetilde{\mathbf{u}}^{-}\right)(\widetilde{\mathbf{A}}-\widetilde{\mathbf{B}})\left(\widetilde{\mathbf{u}}^{+}-\widetilde{\mathbf{u}}^{-}\right) \\
& +\omega\left[\left(\widetilde{\mathbf{u}}^{+}+\widetilde{\mathbf{u}}\right) \widetilde{\mathbf{S}}\left(\widetilde{\mathbf{u}}^{+}-\widetilde{\mathbf{u}}^{-}\right)+1\right] .
\end{aligned}
$$

The variation of $\mathcal{L}_{\text {ex }}$ with respect to $\widetilde{\mathbf{u}}^{ \pm}$gives rise to Eq. (3), and the excitation energy $\omega$ is the stationary points of $\mathcal{L}_{\text {ex }}$. $\widetilde{\mathbf{Z}}$ in Eq. (5) is the well-known $Z$-vector, ${ }^{24}$ and $\delta \mathcal{L}_{\text {ex }} / \delta \widetilde{\mathbf{Z}}=0$ enforces that the orbitals $\widetilde{\psi}$ satisfy the ground state $\mathrm{KS}$ equations and $\Lambda_{i j}=\epsilon_{i j}$. The variation of $\mathcal{L}_{\text {ex }}$ with respect to $\Lambda_{i j}$ results in $\left\langle\widetilde{Z}_{i}|\widetilde{S}| \widetilde{\psi}_{j}\right\rangle=0$, i.e., $\widetilde{Z}_{i}$ should belong to $\Omega^{\text {virt }}$. This is an important condition that will be enforced in Eq. (6). $\partial \mathcal{L}_{\mathrm{ex}} / \partial \Gamma_{i j}=0$ ensures that orbitals $\widetilde{\psi}$ be orthonormal to each other. Finally, $\delta \mathcal{L}_{\mathrm{ex}} / \delta \widetilde{\psi}_{i}=0$ is solved by projections onto both space $\Omega^{\text {virt }}$ and the space spanned by the occupied KS orbitals $\Omega^{\text {occ }}$. The first projection leads to a linear equation

$$
\left[\left(\widetilde{\mathbf{A}}^{\mathrm{S}}+\widetilde{\mathbf{B}}^{\mathrm{S}}\right) \widetilde{\mathbf{Z}}\right]_{i}=-\widetilde{P}_{\mathrm{c}}\left|\frac{\delta \mathcal{L}}{\delta \widetilde{\psi}_{i}}\right\rangle,
$$

which can be solved to obtain $\widetilde{Z}_{i}$. The right-hand side of Eq. (6) is shown in Appendix C. Since $\widetilde{\mathbf{Z}}$ belongs to the full Hilbert space $\Omega_{1}^{\text {virt }} \oplus \Omega_{2}^{\text {virt }} \cdots \oplus \Omega_{N_{\text {occ }}}^{\text {virt }}$, Eq. (6) should be solved in the 
same space; this is because there is one-to-one correspondence between $\widetilde{Z}_{i}$ and $\widetilde{\psi}_{i}$ with the former ensuring the latter to satisfy the ground state KS equation. Note that Eq. (6) can be solved efficiently even in the full space. The second projection yields the solution of $\Gamma_{i j}$ given by

$\left(1+\delta_{i j}\right) \Gamma_{i j}=\left\langle\widetilde{\psi}_{j} \mid \frac{\delta \mathcal{L}}{\delta \widetilde{\psi}_{i}}\right\rangle+\left\langle\widetilde{\psi}_{j} \mid \frac{\delta \sum_{k}\left\langle\widetilde{Z}_{k}\left|\left(\widetilde{H}-\epsilon_{k} \widetilde{S}\right)\right| \widetilde{\psi}_{k}\right\rangle}{\delta \widetilde{\psi}_{i}}\right\rangle$.

Once all variables in the Lagrangian functional are obtained, we can evaluate the ionic forces in excited states as $F_{\text {ex }}=-\partial \mathcal{L}_{\text {ex }} / \partial R$, where

$$
\frac{\partial \mathcal{L}_{\mathrm{ex}}}{\partial R}=\frac{\partial \mathcal{L}}{\partial R}+\sum_{i}\left\langle\widetilde{Z}_{i}\left|\frac{\partial\left(\widetilde{H}-\epsilon_{i} \widetilde{S}\right)}{\partial R}\right| \widetilde{\psi}_{i}\right\rangle-\sum_{i \geq j} \Gamma_{i j}\left\langle\widetilde{\psi}_{i}\left|\frac{\partial \widetilde{S}}{\partial R}\right| \widetilde{\psi}_{j}\right\rangle .
$$

The full expression of $\partial \mathcal{L} / \partial R$ can be found in Appendix D. Note that the last term in Eq. (8) is necessary because $\widetilde{S}$ in the PAW formulation depends explicitly on ionic coordinate $R$ and thus contributes to the ionic forces. This term was absent in Hutter's paper ${ }^{17}$ because the overlap operator $\widetilde{S}$ over there was identity and had no contribution to the forces. The force obtained in Eq. (8) should be combined with the ground state force contribution to arrive at the full expression of the ionic force.

\section{VALIDATIONS}

In the following, we validate the proposed method by applying it to formaldehyde molecule, which is routinely used as a benchmark in TDDFT calculations. The calculations are carried out in the full Hilbert space for the excited state ${ }^{1} A^{\prime \prime}$. The Vienna $a b$ initio simulation package ${ }^{26,27}$ is employed for the TDDFT calculations. The dimensions of the supercell are $20 \AA \times 20 \AA \times 20 \AA$, and the energy cutoff of the planewave basis is $400 \mathrm{eV}$ with the local density approximation $(\mathrm{LDA})^{28}$ for the exchange-correlation (XC) functional. The force convergence criterion is set as $0.05 \mathrm{eV} / \AA$. As shown in Table I, the results from the proposed method agree very well to the conventional TDDFT and experimental values.

To validate the sub-Hilbert space formulation, we calculate the equilibrium geometries and excitation energies of $\mathrm{N}_{2}$ molecule in the singlet excited states ${ }^{1} \Pi_{g}$ and ${ }^{1} \Sigma_{u}^{-}$. The computational parameters are the same as those in formaldehyde. There are two degenerate, occupied $\pi$ orbitals and three occupied $\sigma$ orbitals in $\mathrm{N}_{2}$. The excitation ${ }^{1} \Sigma_{u}^{-}$should only involve the $\pi$ orbitals by a symmetry consideration, which is borne out in the calculations - the energy and geometry are identical between the calculation with the two $\pi$

TABLE I. The calculated equilibrium geometry and excitation energies of formaldehyde in the excited state ${ }^{1} A^{\prime \prime}\left(n \rightarrow \pi^{*}\right)$ as compared to the experiment ("Expt.") and the conventional TDDFT method ("Calc.").

\begin{tabular}{lccccc}
\hline \hline & $R_{\mathrm{CO}}(\AA)$ & $R_{\mathrm{CH}}(\AA)$ & $\iota_{\mathrm{HCH}}\left({ }^{\circ}\right)$ & $\Phi\left(^{\circ}\right)$ & $\Delta E(\mathrm{eV})$ \\
\hline This work & 1.29 & 1.11 & 115.1 & 33 & 3.45 \\
Calc. $^{17}$ & 1.31 & 1.10 & 116.8 & 30 & 3.53 \\
Calc. $^{25}$ & 1.28 & 1.11 & 115.0 & 34 & 3.38 \\
Expt. $^{29}$ & 1.32 & 1.10 & 118.4 & 34 & 3.49 \\
\hline \hline
\end{tabular}

orbitals included $\left(N_{\mathrm{v}}=3\right)$ and the calculation with all occupied orbitals included $\left(N_{\mathrm{v}}=5\right)$. In other words, the sub-Hilbert space calculation for the state ${ }^{1} \Sigma_{u}^{-}$yields the same results as the full Hilbert space calculation. For the excitation ${ }^{1} \Pi_{g}$, on the other hand, all occupied orbitals are involved and should be included for the exact calculation. However, the contributions from the occupied orbitals are not equal and the major contribution comes from the first $\sigma$ orbital $\left(N_{\mathrm{v}}=1\right)$. Overall, the subspace method yields an excellent agreement with the conventional TDDFT method (Table II).

Next, we study two larger systems - a fullerene $\mathrm{C}_{60}$ and an oligothiophenes $\mathrm{T}_{12}$ molecule. The dimensions of the supercell are $24 \AA \times 24 \AA \times 24 \AA$ and $15 \AA \times 20 \AA \times 60 \AA$ for $\mathrm{C}_{60}$ and $\mathrm{T}_{12}$, respectively. Under the ground state geometry, we calculate the excitation energy of eight low-lying excited states and the ionic forces of the first excited state. There are 30 and $24 \pi$ orbitals in $\mathrm{C}_{60}$ and $\mathrm{T}_{12}$, respectively, which are included in the subspace TDDFT calculations because they dominate the low-lying excited states. Fig. 1 illustrates the errors in excitation energy and ionic force as a function of $N_{\mathrm{v}}$ as compared to the "exact" TDDFT values in the full Hilbert space, as well as their computational time. In general, we find that the errors in both energy $(\sim 0.02 \mathrm{eV})$ and force $(\sim 0.06 \mathrm{eV} / \AA)$ are rather small, within the typical convergence criteria in TDDFT energy and force calculations. This is true even if the size of the sub-Hilbert space is $1 / 4$ of the full Hilbert space, in which case the computation time of the present method is comparable to that of the ground state calculation. We have also calculated the oscillator strength as a function of excitation energy for the two systems in the UV/Vis energy range. $N_{\mathrm{v}}=48$ is chosen in these calculations. As shown in Fig. 1, both the excitation energies and oscillator strengths show good agreement between the present method and the well-established Gaussian method ${ }^{30}$ with $6-31 G^{*}$ basis set and LDA functional.

We also perform Born-Oppenheimer Molecular Dynamics (BOMD) simulations of $\mathrm{C}_{60}$ and $\mathrm{T}_{12}$ in their lowest excited state to further validate the sub-Hilbert space formulation. Four low-lying excited states are included in the TDDFT calculations at each BOMD time step, and $N_{\mathrm{v}}=48$ is included in the sub-Hilbert space. We first relax the molecular geometry at $0 \mathrm{~K}$ in the lowest excited state with a force criterion of $0.05 \mathrm{eV} / \AA$. Subsequently, the system is brought to $300 \mathrm{~K}$ by using a repeated velocity scaling and then kept at $300 \mathrm{~K}$ with a thermal bath for $200 \mathrm{fs}$. Finally, a micro-canonical production run is carried out for 500 time steps with the time step of $1 \mathrm{fs}$.

TABLE II. The equilibrium bond length $\left(R_{\mathrm{NN}}\right.$ in $\AA$ ) and excitation energies ( $\triangle E$ in eV) of $\mathrm{N}_{2}$ as a function of $N_{\mathrm{v}}$ in the excited states ${ }^{1} \Sigma_{u}^{-}$and ${ }^{1} \Pi_{g}$. $N_{\mathrm{v}}=5$ corresponds to the "exact" conventional calculation. For comparison, the values of $R_{\mathrm{NN}}$ and $\Delta E$ calculated by using a Gaussian basis set ${ }^{25}$ are also presented.

\begin{tabular}{lcccccc}
\hline \hline State & & $N_{\mathrm{v}}=1$ & $N_{\mathrm{v}}=3$ & $N_{\mathrm{v}}=4$ & $N_{\mathrm{v}}=5$ & Ref. 25 \\
\hline${ }^{1} \Sigma_{u}^{-}$ & $R_{\mathrm{NN}}$ & N/A & 1.28 & 1.28 & 1.28 & 1.27 \\
& $\Delta E$ & N/A & 8.14 & 8.14 & 8.14 & 8.27 \\
${ }^{1} \Pi_{g}$ & $R_{\mathrm{NN}}$ & 1.20 & 1.21 & 1.21 & 1.22 & 1.21 \\
& $\Delta E$ & 8.54 & 8.47 & 8.42 & 8.38 & 8.46 \\
\hline \hline
\end{tabular}



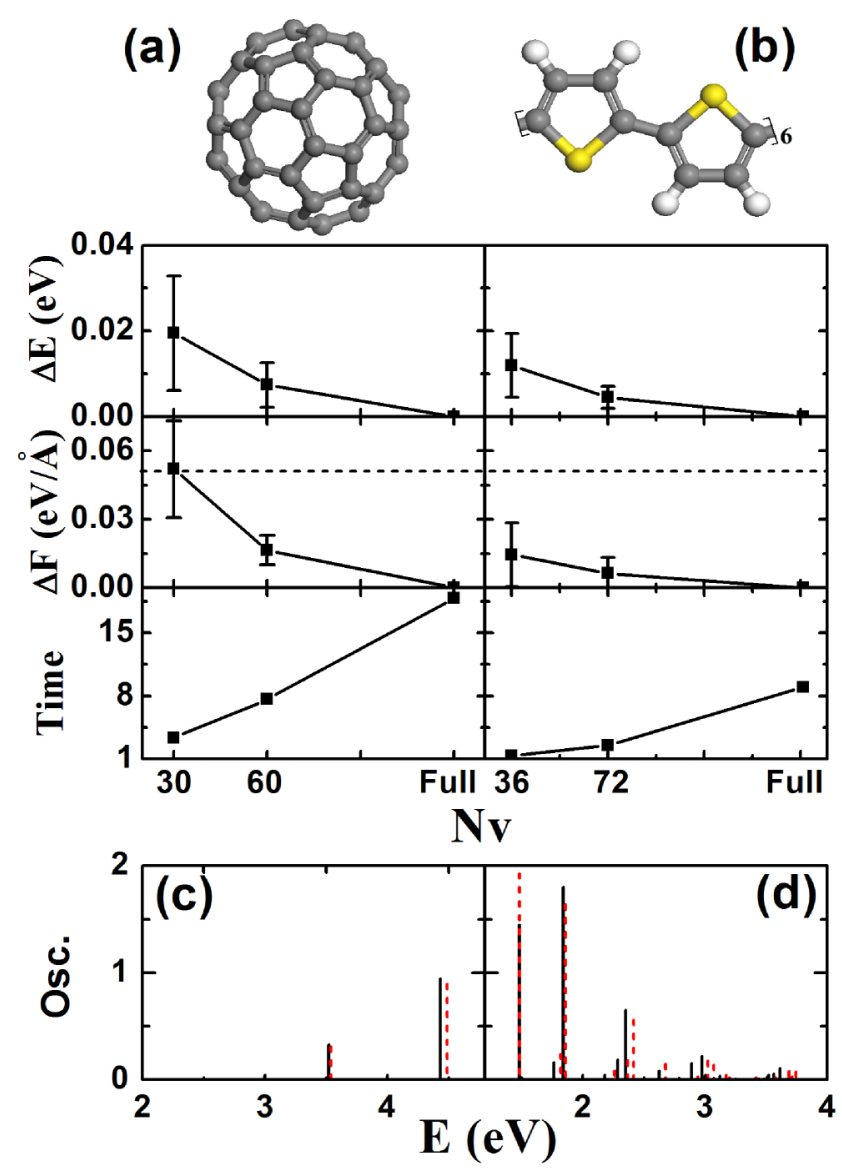

FIG. 1. Average errors in the excitation energy and ionic force, as well as the computational time as a function of $N_{\mathrm{v}}$ for (a) $\mathrm{C}_{60}$ and (b) $\mathrm{T}_{12}$. "Full" indicates the calculations in the full Hilbert space with $N_{\mathrm{v}}=N_{\text {occ }}$, where $N_{\text {occ }}=120$ and 145 for $\mathrm{C}_{60}$ and $\mathrm{T}_{12}$, respectively. Error bars denote the standard deviations. The computational time of the ground state calculation is set to unity. The horizontal dashed line indicates the typical force convergence criterion in geometry relaxations. The oscillator strength as a function of excitation energy for the low-lying dipole-allowed-excitations of (c) $\mathrm{C}_{60}$ and (d) $T_{12}$. Black solid and red dashed lines indicate the values obtained with the present approach and the GAUSSIAN09 software, respectively.

In Fig. 2, we plot the total energy and its break-up as a function of BOMD time step for both $\mathrm{C}_{60}$ and $\mathrm{T}_{12}$. It is evident that the dynamics is stable and the total energy is conserved with the maximum energy fluctuation of $0.09 \mathrm{eV}$ and $0.25 \mathrm{eV}$ for $\mathrm{C}_{60}$ and $\mathrm{T}_{12}$, respectively. These energy fluctuations are similar to those in the ground state BOMD simulations. The larger energy fluctuation in $T_{12}$ is due to the presence of protons and can be reduced by decreasing the BOMD time step. Note that this represents a rigorous test for the subspace TDDFT method because if the energy or force were not calculated sufficiently accurate, the dynamics would not be stable and the total energy would diverge.

Finally, we apply the subspace TDDFT method to the lowest excited state in disordered P3HT conjugated polymers which are used as donor materials in organic photovoltaics. The supercell has a dimension of $18.2 \AA$, consisted of 606 atoms with three P3HT chains and twenty-four thiophene rings as shown in Fig. 3. For this system, $N_{\mathrm{v}}=24$ is found to be sufficient to yield converged values for both excitation energy and ionic forces. The energy of the lowest excited state is $1.7 \mathrm{eV}$, comparing well to the experimental value of

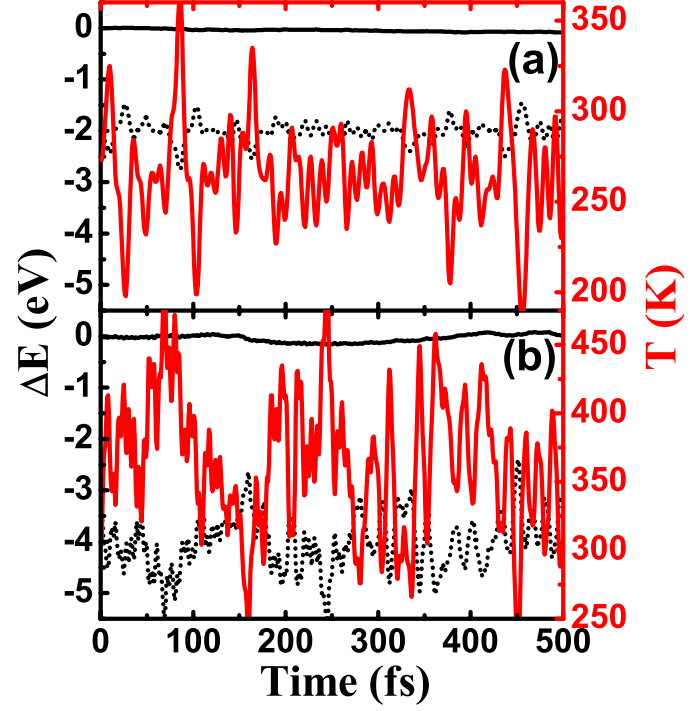

FIG. 2. The total energy (black solid), potential energy (black dashed), and temperature (red solid) as a function of time in the micro-canonical MD simulations of $\mathrm{C}_{60}$ (a) and $\mathrm{T}_{12}$ (b). $\Delta E$ denotes the energy relevant to the total energy at time zero.

$\sim 2.0 \mathrm{eV} .{ }^{31}$ Moreover, the computation time is only $50 \%$ of that of the ground state calculation, demonstrating the potential of the method for dealing with even larger systems. The similar error analysis as presented in Fig. 1 is performed and included in Fig. 3.

\section{DISCUSSION}

Here, we analyze computational scaling in the solution of TDKS eigenvalue equations, which is the third bottleneck to be overcome. The computationally most demanding step is to calculate $\widetilde{\mathbf{A}} \widetilde{\mathbf{u}}$ and $\widetilde{\mathbf{B}} \widetilde{\mathbf{u}}$. As shown in Eq. (4), two matrix multiplication terms are involved: the first one has to do with the KS Hamiltonian acting on orbitals, and the second one evaluates the TDDFT kernels. For the first term, the computational time $t_{\text {full }}^{\mathrm{H}}$ scales as $N_{\mathrm{occ}} N_{\mathrm{plw}} \ln N_{\text {plw }}$ in the fullspace formulation, where $N_{\text {plw }}$ is the number of plane-wave basis, and $N_{\mathrm{plw}} \ln N_{\mathrm{plw}}$ corresponds to the computational cost of FFT. In the calculation of the kernels, the most timeconsuming step involves the Gram-Schmidt orthonormalization whose computational time $t_{\text {full }}^{\mathrm{K}}$ scales as $N_{\mathrm{occ}}^{2} \times N_{\mathrm{plw}}$. In the present subspace TDDFT method, the occupied manifold is truncated and the dimension of the reduced sub-Hilbert space is $N_{\mathrm{v}} \times N_{\text {virt }}$. Hence, the corresponding computational time is given by $t_{\text {sub }}^{\mathrm{H}} \sim N_{\mathrm{v}} N_{\text {plw }} \ln N_{\text {plw }}$ and $t_{\text {sub }}^{\mathrm{K}} \sim N_{\mathrm{v}} \times N_{\text {occ }} \times N_{\text {plw }}$. Therefore, the computational time of the subspace method relative to the full Hilbert space method scales as $N_{\mathrm{v}} / N_{\text {occ }}$ $(\approx 0.1-0.3)$. In practice, we find that the computational scaling of the subspace method is even more favorable than $N_{\mathrm{v}} / N_{\text {occ }}$ as shown in Fig. 1. This probably stems from a smaller number of iteration steps and reduced communications between central processing units in parallel calculations.

Next we discuss why the subspace TDDFT method could yield the similar accuracy as the conventional TDDFT method for both energy and force. An excited state can be viewed as a linear combination of transitions from an occupied orbital 


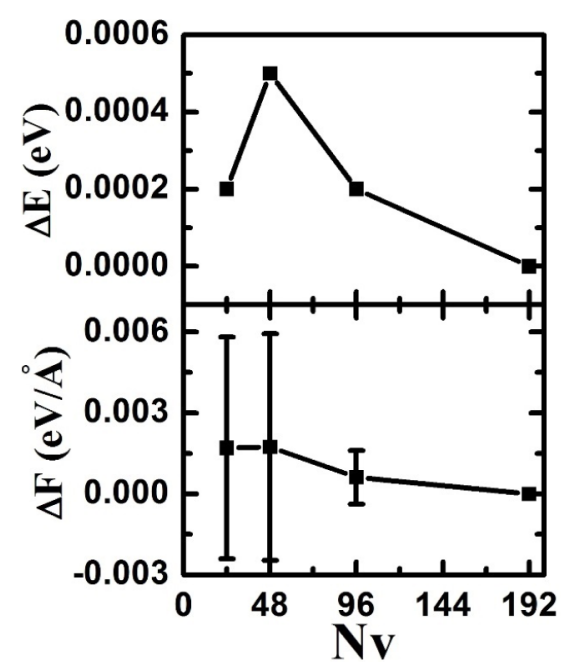

to a virtual orbital, $i \rightarrow a$, and the relative contribution of the transition to the excited state scales roughly as $1 /\left(\epsilon_{a}-\epsilon_{i}-\omega\right)$. Hence, the closer the KS transition energy to the excitation energy $\omega$, the greater the contribution of the transition. Very often, the excited state can be approximated very well by only a few such transitions. ${ }^{32}$ Thus, a truncation of (occupied or virtual) orbitals is often justified. This is the reason why both the subspace TDDFT method (truncating occupied orbitals) and Doltsinis's method (truncating virtual orbitals) could yield a reasonable approximation to the excitation energy. On the other hand, the force calculation involves variation of KS orbitals with respect to ionic displacement, which depends crucially on the completeness of the KS orbitals as demonstrated by Doltsinis and Kosov. ${ }^{10}$ Therefore, any truncation of full KS space would be prone to errors in force calculations. In the present method, the variation of the KS orbitals is captured by the $Z$-vector thanks to the Lagrangian formulation, and $\widetilde{\mathbf{Z}}$ is evaluated in the full Hilbert space through Eq. (6) with a complete set of KS orbitals. Therefore, the subspace TDDFT method could yield the similar accuracy as the conventional TDDFT for the force calculations.

\section{CONCLUSIONS AND OUTLOOKS}

To summarize, we have developed an efficient TDDFT method to compute the excitation energy and most importantly, the ionic forces for large systems using plane-waves and pseudopotentials. The method is based on the density functional perturbation theory which renders a clean separation of Hilbert spaces for the solution of TDKS eigenvalue equations. The key bottleneck of the conventional TDDFT method is circumvented by projecting the TDKS equations from a full Hilbert space to a substantially reduced sub-Hilbert space. The method is validated for several model systems and exhibits the similar accuracy as the conventional TDDFT method in the full Hilbert space, but at a computational cost of the ground state calculation. Finally, we show that the Born-Oppenheimer molecular dynamics can be successfully performed for excited states with the present method, which paves ways to many applications that are presently beyond the reach of TDDFT methods.
FIG. 3. Left: Average errors in the excitation energy and ionic force as a function of $N_{\mathrm{v}}$ for the lowest excited state of P3HT. Right: Atomic structure of the disorder P3HT polymers and the charge density difference between the lowest excited state and the ground state. The blue (yellow) iso-surfaces illustrate the charge density difference at $+(-) 0.002 \AA^{-3}$. The gray, white, and yellow spheres denote $\mathrm{C}, \mathrm{H}$, and $\mathrm{S}$ atoms, respectively.
Two important extensions of the method can be envisioned. A hybrid XC functional or a range-separated hybrid $\mathrm{XC}$ functional ${ }^{33-35}$ can be implemented into the method; the latter is particularly useful to tackle Rydberg states, ${ }^{36}$ chargetransfer excitations, ${ }^{37,38}$ and excitations in weakly bonded complexes. ${ }^{39,40}$ These hybrid functionals are crucial to many applications, especially in extended systems. The hybrid functionals can be easily implemented into the present method, but their computational costs will be substantially higher than the local XC functionals. The most time-consuming part is the determination of the KS Hamiltonian and the kernel functional in Eq. (4).

Another extension has to do with non-adiabatic molecular dynamics for excited states, which is crucial to describe molecular dynamics with electronic transitions. ${ }^{41-43}$ We can calculate the non-adiabatic coupling between excited states by the present method in conjunction with the fewest-switchessurface-hopping algorithm ${ }^{44,45}$ to determine quantum transition probabilities.

\section{ACKNOWLEDGMENTS}

This work was supported by the Office of Naval Research.

\section{APPENDIX A: PAW METHOD}

Here, we briefly summarize the PAW formalism to introduce the quantities that will be used in Appendices B-D. In the PAW method, $\left|\psi_{i}\right\rangle$ is linearly related to $\left|\widetilde{\psi}_{i}\right\rangle$ by

$$
\left|\psi_{i}\right\rangle=\left|\widetilde{\psi}_{i}\right\rangle+\sum_{I}\left(\left|\phi_{I}\right\rangle-\left|\widetilde{\phi}_{I}\right\rangle\right)\left\langle\widetilde{p}_{I} \mid \widetilde{\psi}_{i}\right\rangle,
$$

where $\phi_{I}, \widetilde{\phi}_{I}$, and $\widetilde{p}_{I}$ are the AE partial waves, PS partial waves and projector functions, respectively, within the core. Here, index $I$ also includes the angular momentum quantum numbers and an additional index for the reference energy. The AE charge density related to orbitals $i$ and $j$ is calculated by

$$
\rho_{i j}^{\mathrm{AE}}(r)=\psi_{i}^{*}(r) \psi_{j}(r)=\widetilde{\rho}_{i j}(r)-\widetilde{\rho}_{i j}^{1}(r)+\rho_{i j}^{1}(r),
$$

where the constituent charge densities are defined as

$$
\widetilde{\rho}_{i j}(r)=\widetilde{\psi}_{i}^{*}(r) \widetilde{\psi}_{j}(r),
$$




$$
\begin{aligned}
& \tilde{\rho}_{i j}^{1}(r)=\sum_{I J}\left\langle\widetilde{\psi}_{i} \mid \widetilde{p}_{I}\right\rangle\left\langle\widetilde{p}_{J} \mid \widetilde{\psi}_{j}\right\rangle \widetilde{\phi}_{I}^{*}(r) \widetilde{\phi}_{J}(r), \\
& \rho_{i j}^{1}(r)=\sum_{I J}\left\langle\widetilde{\psi}_{i} \mid \widetilde{p}_{I}\right\rangle\left\langle\widetilde{p}_{J} \mid \widetilde{\psi}_{j}\right\rangle \phi_{I}^{*}(r) \phi_{J}(r) .
\end{aligned}
$$

Here, $\widetilde{\rho}$ is the PS charge density calculated on a plane-wave (uniform) grid. $\widetilde{\rho}^{1}$ and $\rho^{1}$ are one-center charge densities computed on a radial grid within the core around each ion. To treat the long-range electrostatic interactions between the core charge density and PS charge density, a compensation charge density $\hat{\rho}$ on the plane-wave grid is introduced as

$$
\hat{\rho}_{i j}(r)=\sum_{I J, L M}\left\langle\widetilde{\psi}_{i} \mid \widetilde{p}_{I}\right\rangle\left\langle\widetilde{p}_{J} \mid \widetilde{\psi}_{j}\right\rangle \hat{Q}_{I J}^{L M}(r),
$$

where the function $\hat{Q}_{I J}^{L M}(r)$ can be found in Eq. (27) of Ref. 22. $\widetilde{\rho}_{i j}^{1}+\hat{\rho}_{i j}$ has the same moments as the exact density $\rho_{i j}^{1}$ within the core, i.e., the electrostatic potential stemming from $\rho_{i j}^{1}$ is identical to that of $\widetilde{\rho}_{i j}^{1}+\hat{\rho}_{i j}$ outside the cores. Thus, the electrostatic interaction of the core-pseudo and pseudo-pseudo charge densities can be evaluated exactly based on the charge density $\rho_{i j}(r) \equiv \widetilde{\rho}_{i j}(r)+\hat{\rho}_{i j}(r)$. However, the electrostatic interaction of the core-core should be corrected on the radial grid within the core. ${ }^{22}$ The expression of the total energy is conveniently split up to three terms as ${ }^{21,22}$

$$
E=\widetilde{E}+E^{1}-\widetilde{E}^{1},
$$

where $\widetilde{E}$ is evaluated on the plane-wave grid; $E^{1}$ and $\widetilde{E}^{1}$ are calculated on the radial grid. Their detailed expressions can be found in Ref. 22. Based on the total energy, the PS KS Hamiltonian can be obtained by

$$
\begin{aligned}
\widetilde{H} & =\frac{\delta E}{\delta \widetilde{\rho}}=-\frac{1}{2} \nabla^{2}+\widetilde{V}_{\mathrm{eff}} \\
& =-\frac{1}{2} \nabla^{2}+\widetilde{v}_{\mathrm{eff}}(r)+\sum_{I J}\left|\widetilde{p}_{I}\right\rangle\left(\hat{D}_{I J}+D_{I J}^{1}-\widetilde{D}_{I J}^{1}\right)\left\langle\widetilde{p}_{J}\right|,
\end{aligned}
$$

where $\widetilde{\rho}=\sum_{i}\left|\widetilde{\psi}_{i}\right\rangle\left\langle\widetilde{\psi}_{i}\right|$ is the PS density operator. $\widetilde{v}_{\text {eff }}$ is the KS effective potential based on the charge density $\rho_{i j}$, which is evaluated on the plane-wave grid. $\hat{D}_{I J}, D_{I J}^{1}$, and $\widetilde{D}_{I J}^{1}$ are calculated by $\partial \widetilde{E} / \partial \rho_{I J}, \partial E^{1} / \partial \rho_{I J}$, and $\partial \widetilde{E}^{1} / \partial \rho_{I J}$, respectively, where $\rho_{I J}=\sum_{i}\left\langle\widetilde{\psi}_{i} \mid \widetilde{p}_{I}\right\rangle\left\langle\widetilde{p}_{J} \mid \widetilde{\psi}_{i}\right\rangle$ is the occupancy of each augmentation channel $(I, J)$.

\section{APPENDIX B: KERNEL FUNCTIONAL}

The kernel functional $\widetilde{K}$ is calculated by

$$
\begin{aligned}
\widetilde{K} \equiv & \frac{\delta \widetilde{V}_{\mathrm{eff}}}{\delta \widetilde{\rho}}=\int \frac{\delta \widetilde{V}_{\mathrm{eff}}}{\delta \widetilde{n}(r)} \frac{\partial \widetilde{n}(r)}{\partial \widetilde{\rho}} d r+\sum_{I, J} \frac{\partial \widetilde{V}_{\mathrm{eff}}}{\partial \rho_{I J}} \frac{\partial \rho_{I J}}{\partial \widetilde{\rho}} \\
= & \int \frac{\delta\left[\widetilde{v}_{\mathrm{eff}}+\sum_{I J}\left|\widetilde{p}_{I}\right\rangle \hat{D}_{I J}\left\langle\widetilde{p}_{J}\right|\right]}{\delta \widetilde{n}(r)}|r\rangle\langle r| d r \\
& +\sum_{I^{\prime} J^{\prime}, L M} \int \frac{\delta\left[\widetilde{v}_{\mathrm{eff}}+\sum_{I J}\left|\widetilde{p}_{I}\right\rangle \hat{D}_{I J}\left\langle\widetilde{p}_{J}\right|\right]}{\delta \hat{n}(r)} \\
& \times \hat{Q}_{I^{\prime} J^{\prime}}^{L M}(r)\left|\widetilde{p}_{I^{\prime}}\right\rangle\left\langle\widetilde{p}_{J^{\prime}}\right| d r \\
& +\sum_{I^{\prime} J^{\prime}} \frac{\partial \sum_{I J}\left|\widetilde{p}_{I}\right\rangle\left(D_{I J}^{1}-\widetilde{D}_{I J}^{1}\right)\left\langle\widetilde{p}_{J}\right|}{\partial \rho_{I^{\prime} J^{\prime}}}\left|\widetilde{p}_{I^{\prime}}\right\rangle\left\langle\widetilde{p}_{J^{\prime}}\right|,
\end{aligned}
$$

where $\tilde{n}(r) \equiv \sum_{i} \tilde{\rho}_{i i}(r)$ and $\hat{n}(r) \equiv \sum_{i} \hat{\rho}_{i i}(r)$. The spin indices have been omitted. We also define $n(r) \equiv \tilde{n}(r)+\hat{n}(r)$ as the total charge density on the plane-wave grid. The matrix elements of the kernel in terms of KS orbitals $\widetilde{K}_{i j, k l} \equiv\left\langle\widetilde{\psi}_{i} \widetilde{\psi}_{j}|\widetilde{K}| \widetilde{\psi}_{k} \widetilde{\psi}_{l}\right\rangle$ are given by

$$
\begin{aligned}
\widetilde{K}_{i j, k l}= & \int \frac{\rho_{i j}(r) \rho_{k l}(r)}{\left|r-r^{\prime}\right|} d r d r^{\prime}+\int \rho_{i j}(r) \frac{\delta^{2} E_{\mathrm{xc}}}{\delta n^{2}} \rho_{k l}(r) d r \\
& +\sum_{I J, I^{\prime} J^{\prime}}\left\langle\tilde{\psi}_{i} \mid \tilde{p}_{I}\right\rangle\left\langle\tilde{p}_{J} \mid \tilde{\psi}_{j}\right\rangle \frac{\delta^{2}\left(E^{1}-\tilde{E}^{1}\right)}{\delta \rho_{I J} \delta \rho_{I^{\prime} J^{\prime}}}\left\langle\tilde{\psi}_{k} \mid \tilde{p}_{I^{\prime}}\right\rangle\left\langle\tilde{p}_{J^{\prime}} \mid \tilde{\psi}_{l}\right\rangle .
\end{aligned}
$$

\section{APPENDIX C: LAGRANGIAN DERIVATIVES}

To show the projection of $\delta \mathcal{L} / \delta \widetilde{\psi}_{i}$ onto the space $\Omega^{\text {virt }}$, we first define several equations

$$
\begin{aligned}
\widetilde{M}_{i} & =\widetilde{u}_{i}^{+}+\widetilde{u}_{i}^{-}, \\
\widetilde{L}_{i} & =\widetilde{u}_{i}^{+}-\widetilde{u}_{i}^{-}, \\
\Delta \widetilde{\rho}[\widetilde{\mathbf{u}}](r) & =\sum_{i} \widetilde{\psi}_{i}(r) \widetilde{u}_{i}(r), \\
\Delta \widetilde{\rho}^{(\mathrm{x})}(r)= & \frac{1}{2} \sum_{i} \widetilde{M}_{i}(r) \widetilde{M}_{i}(r)+\frac{1}{2} \sum_{i} \widetilde{L}_{i}(r) \widetilde{L}_{i}(r) \\
& -\frac{1}{2} \sum_{i j} \widetilde{\psi}_{i}(r) \widetilde{\psi}_{j}(r)\left(\left\langle\widetilde{M}_{i}\left|\widetilde{S}_{\mid}\right| \widetilde{M}_{j}\right\rangle+\left\langle\widetilde{L}_{i}|\widetilde{S}| \widetilde{L}_{j}\right\rangle\right) .
\end{aligned}
$$

The corresponding expression in the real space is

$$
\begin{aligned}
\left\langle r\left|\widetilde{P}_{\mathrm{c}}\right| \frac{\delta \mathcal{L}}{\delta \widetilde{\psi}_{i}}\right\rangle= & 2 \int \widetilde{\psi}_{i}(r) \widetilde{K}^{\mathrm{S}}\left(r, r^{\prime}\right) \Delta \widetilde{\rho}^{(\mathrm{x})}\left(r^{\prime}\right) d r^{\prime}-2 \sum_{j} \widetilde{S}_{j}(r) \int \widetilde{\psi}_{i}\left(r^{\prime}\right) \widetilde{\psi}_{j}\left(r^{\prime}\right) \widetilde{K}^{\mathrm{S}}\left(r^{\prime}, r^{\prime \prime}\right) \Delta \widetilde{\rho}^{(\mathrm{x})}\left(r^{\prime \prime}\right) d r^{\prime} d r^{\prime \prime} \\
& +2 \int \widetilde{M}_{i}(r) \widetilde{K}^{\mathrm{S}}\left(r, r^{\prime}\right) \Delta \widetilde{\rho}[\widetilde{\mathbf{M}}]\left(r^{\prime}\right) d r^{\prime}-2 \sum_{j} \widetilde{S} \widetilde{\psi}_{j}(r) \int \widetilde{M}_{i}\left(r^{\prime}\right) \widetilde{\psi}_{j}\left(r^{\prime}\right) \widetilde{K}^{\mathrm{S}}\left(r^{\prime}, r^{\prime \prime}\right) \Delta \widetilde{\rho}[\widetilde{\mathbf{M}}]\left(r^{\prime \prime}\right) d r^{\prime} d r^{\prime \prime} \\
& -2 \sum_{j} \widetilde{S}_{j}(r) \int \widetilde{\psi}_{i}\left(r^{\prime}\right) \widetilde{\psi}_{j}\left(r^{\prime}\right) \widetilde{K}^{\mathrm{S}}\left(r^{\prime}, r^{\prime \prime}\right) \Delta \widetilde{\rho}[\widetilde{\mathbf{M}}]\left(r^{\prime \prime}\right) d r^{\prime} d r^{\prime \prime}+2 \int \widetilde{\psi}_{i}(r) \widetilde{K}^{\mathrm{S}^{\prime}}\left(r, r^{\prime}, r^{\prime \prime}\right) \Delta \widetilde{\rho}[\widetilde{\mathbf{M}}]\left(r^{\prime}\right) \Delta \widetilde{\rho}[\widetilde{\mathbf{M}}]\left(r^{\prime \prime}\right) d r^{\prime} d r^{\prime \prime} \\
& -2 \sum_{j} \widetilde{S}_{j}(r) \int \widetilde{\psi}_{i}\left(r^{\prime}\right) \widetilde{\psi}_{j}\left(r^{\prime}\right) \widetilde{K}^{\mathrm{S}^{\prime}}\left(r^{\prime}, r^{\prime \prime}, r^{\prime \prime \prime}\right) \Delta \widetilde{\rho}[\widetilde{\mathbf{M}}]\left(r^{\prime \prime}\right) \times \Delta \widetilde{\rho}[\widetilde{\mathbf{M}}]\left(r^{\prime \prime \prime}\right) d r^{\prime} d r^{\prime \prime} d r^{\prime \prime \prime},
\end{aligned}
$$

where $\widetilde{K}^{\mathrm{S}^{\prime}} \equiv \delta \widetilde{K}^{\mathrm{S}} / \delta \widetilde{\rho}$ includes the third derivative of the functionals $E_{\mathrm{xc}}$ and $\left(E^{1}-\tilde{E}^{1}\right)$. The projection of $\delta \mathcal{L} / \delta \widetilde{\psi}_{i}$ onto the space $\Omega^{\text {occ }}$, i.e., $\left\langle\widetilde{\psi}_{j} \mid \delta \mathcal{L} / \delta \widetilde{\psi}_{i}\right\rangle$ can be obtained similarly. The second term at the right hand side in Eq. (7) can be calculated by 


$$
2 \int \widetilde{\psi}_{i}(r) \widetilde{\psi}_{j}(r) \widetilde{K}^{\mathrm{S}}\left(r, r^{\prime}\right) \Delta \widetilde{\rho}[\widetilde{\mathbf{Z}}]\left(r^{\prime}\right) d r d r^{\prime} .
$$

We use the finite difference method to calculate the second and third derivatives of the XC energy functional $E_{\mathrm{xc}}$ as

$$
\int \frac{\delta^{2} E_{\mathrm{xc}}}{\delta n(r) \delta n\left(r^{\prime}\right)} \ln _{n} \rho\left(r^{\prime}\right) d r^{\prime} \approx \sum_{k} \frac{a_{k}}{2 \Delta} V_{\mathrm{xc}}(n+k \Delta \rho)
$$

for the second derivatives, and $a_{k}=1,0,1$ for $k=-1,0,1$ with a three-point formula. For the third derivatives, we have

$$
\begin{gathered}
\int \frac{\delta^{3} E_{\mathrm{xc}}}{\delta n(r) \delta n\left(r^{\prime}\right) \delta n\left(r^{\prime \prime}\right)} \ln \rho\left(r^{\prime}\right) \rho\left(r^{\prime \prime}\right) d r^{\prime} d r^{\prime \prime} \\
\approx \sum_{k} \frac{a_{k}}{\Delta^{2}} V_{\mathrm{xc}}(n+k \Delta \rho),
\end{gathered}
$$

where $a_{k}=1,-2,1$ for $k=-1,0,1$. Numerical tests have shown that stable results can be achieved by using $\Delta=10^{-2}$. The second and third derivatives of $\left(E^{1}-\tilde{E}^{1}\right)$ can be calculated similarly using the finite difference method.

\section{APPENDIX D: FORCE}

The variation of Lagrangian $\mathcal{L}$ with respect to the ionic coordinate $R$ is calculated by

$$
\begin{aligned}
\frac{\partial \mathcal{L}}{\partial R}= & \frac{1}{2} \sum_{i}\left[\left\langle\widetilde{M}_{i}\left|\frac{\partial\left(\widetilde{H}-\epsilon_{i} \widetilde{S}\right)}{\partial R}\right| \widetilde{M}_{i}\right\rangle+\left\langle\widetilde{L}_{i}\left|\frac{\partial\left(\widetilde{H}-\epsilon_{i} \widetilde{S}\right)}{\partial R}\right| \widetilde{L}_{i}\right\rangle\right]-\frac{1}{2} \sum_{i j}\left\langle\widetilde{\psi}_{i}\left|\frac{\partial \widetilde{H}}{\partial R}\right| \widetilde{\psi}_{j}\right\rangle\left(\left\langle\widetilde{M}_{i}|\widetilde{S}| \widetilde{M}_{j}\right\rangle+\left\langle\widetilde{L}_{i}|\widetilde{S}| \widetilde{L}_{j}\right\rangle\right) \\
& +\omega \sum_{i}\left\langle\widetilde{M}_{i}\left|\frac{\partial \widetilde{S}}{\partial R}\right| \widetilde{L}_{i}\right\rangle+2 \int \frac{\partial \Delta \widetilde{\rho}[\widetilde{\mathbf{M}}](r)}{\partial R} \widetilde{K}^{\mathrm{S}}\left(r, r^{\prime}\right) \Delta \widetilde{\rho}[\widetilde{\mathbf{M}}]\left(r^{\prime}\right) d r d r^{\prime} \\
& -2 \sum_{i j}\left\langle\widetilde{\psi}_{i}\left|\frac{\partial \widetilde{S}}{\partial R}\right| \widetilde{M}_{j}\right\rangle \int \widetilde{\psi}_{i}(r) \widetilde{\psi}_{j}(r) \widetilde{K}^{\mathrm{s}}\left(r, r^{\prime}\right) \Delta \widetilde{\rho}[\widetilde{\mathbf{M}}]\left(r^{\prime}\right) d r d r^{\prime} \\
& +2 \sum_{i} \int \frac{\partial \rho_{i i}(r)}{\partial R} \widetilde{K}^{\mathrm{S}^{\prime}}\left(r, r^{\prime}, r^{\prime \prime}\right) \Delta \widetilde{\rho}[\widetilde{\mathbf{M}}]\left(r^{\prime}\right) \Delta \widetilde{\rho}[\widetilde{\mathbf{M}}]\left(r^{\prime \prime}\right) d r d r^{\prime} d r^{\prime \prime} .
\end{aligned}
$$

In the PAW method, the partial derivative of the Hamiltonian with respect to $R$ is more complicated due to the explicit dependence of the projector functions on $R$. The partial derivatives of KS Hamiltonian $H_{i j}$ elements with respect to ionic position are expressed as

$$
\begin{aligned}
& \frac{\partial H_{i j}}{\partial R}=\int \rho_{i j}(r) \frac{\partial \widetilde{v}_{\mathrm{eff}}(r)}{\partial R} d r+\sum_{I J} \frac{\partial\left\langle\widetilde{\psi}_{i} \mid \widetilde{p}_{I}\right\rangle\left\langle\widetilde{p}_{J} \mid \widetilde{\psi}_{j}\right\rangle}{\partial R}\left(\hat{D}_{I J}+D_{I J}^{1}-\widetilde{D}_{I J}^{1}\right)+\sum_{I J}\left\langle\widetilde{\psi}_{i} \mid \widetilde{p}_{I}\right\rangle\left\langle\widetilde{p}_{J} \mid \widetilde{\psi}_{j}\right\rangle\left[\left.\frac{\partial \hat{D}_{I J}}{\partial R}\right|_{\widetilde{v}_{\mathrm{eff}}}+\frac{\partial\left(D_{I J}^{1}-\widetilde{D}_{I J}^{1}\right)}{\partial R}\right] \\
& =\int \rho_{i j}(r) \frac{\partial v_{\mathrm{H}}\left[\widetilde{n}_{Z c}\right]}{\partial R} d r+2 \int v_{\mathrm{H}}\left[\rho_{i j}\right] \sum_{k} \frac{\partial \rho_{k k}(r)}{\partial R} d r+\int \rho_{i j}(r)\left[\frac{\delta^{2} E_{\mathrm{xc}}}{\delta n^{\uparrow} \delta n^{\uparrow}}+\frac{\delta^{2} E_{\mathrm{xc}}}{\delta n^{\uparrow} \delta n^{\downarrow}}\right] \sum_{k} \frac{\partial \rho_{k k}(r)}{\partial R} d r \\
& +\sum_{I J} \frac{\partial\left\langle\widetilde{\psi}_{i} \mid \widetilde{p}_{I}\right\rangle\left\langle\widetilde{p}_{J} \mid \widetilde{\psi}_{j}\right\rangle}{\partial R}\left(\hat{D}_{I J}+D_{I J}^{1}-\widetilde{D}_{I J}^{1}\right)+\left.\sum_{I J}\left\langle\widetilde{\psi}_{i} \mid \widetilde{p}_{I}\right\rangle\left\langle\widetilde{p}_{J} \mid \widetilde{\psi}_{j}\right\rangle \frac{\partial \hat{D}_{I J}}{\partial R}\right|_{\tilde{v}_{\mathrm{eff}}} \\
& +\sum_{I J, I^{\prime} J^{\prime}}\left\langle\widetilde{\psi}_{i} \mid \widetilde{p}_{I}\right\rangle\left\langle\widetilde{p}_{J} \mid \widetilde{\psi}_{j}\right\rangle\left[\frac{\delta^{2}\left(E^{1}-\widetilde{E}^{1}\right)}{\delta \rho_{I J}^{\uparrow} \delta \rho_{I^{\prime} J^{\prime}}^{\uparrow}}+\frac{\delta^{2}\left(E^{1}-\widetilde{E}^{1}\right)}{\delta \rho_{I J}^{\uparrow} \delta \rho_{I^{\prime} J^{\prime}}^{\downarrow}}\right] \sum_{k} \frac{\partial\left\langle\widetilde{\psi}_{k} \mid \widetilde{p}_{I^{\prime}}\right\rangle\left\langle\widetilde{p}_{J^{\prime}} \mid \widetilde{\psi}_{k}\right\rangle}{\partial R},
\end{aligned}
$$

where $v_{\mathrm{H}}[\rho]$ is the Coulomb potential of charge density $\rho$ and $v_{\mathrm{H}}\left[\widetilde{n}_{Z c}\right]$ represents the ionic pseudopotential due to the pseudocore charge density $\widetilde{n}_{Z c}$.

${ }^{1}$ E. Runge and E. K. U. Gross, Phys. Rev. Lett. 52, 997 (1984).

${ }^{2}$ M. A. L. Marques and E. K. U. Gross, Annu. Rev. Phys. Chem. 55, 427 (2004).

${ }^{3}$ M. E. Casida, in Recent Advances in Density Functional Methods, edited by

D. P. Chong (World Scientific, Singapore, 1995), pp. 155-192.

${ }^{4}$ M. Petersilka, U. J. Gossmann, and E. K. U. Gross, Phys. Rev. Lett. 76, 1212 (1996).

${ }^{5}$ R. Bauernschmitt and R. Ahlrichs, Chem. Phys. Lett. 256, 454 (1996).

${ }^{6}$ I. Vasiliev, S. Ogut, and J. R. Chelikowsky, Phys. Rev. Lett. 82, 1919 (1999).

${ }^{7}$ B. Walkera and R. Gebauer, J. Chem. Phys. 127, 164106 (2007).
${ }^{8}$ M. E. Casida and M. Huix-Rotllant, Annu. Rev. Phys. Chem. 63, 287323 (2012).

${ }^{9}$ F. Furche and K. Burke, Annu. Rep. Comput. Chem. 1, 19 (2005).

${ }^{10}$ N. Doltsinis and D. Kosov, J. Chem. Phys. 122, 144101 (2005).

${ }^{11}$ M. Walter, H. Hakkinen, L. Lehtovaara, M. Puska, J. Enkovaara, C. Rostgaard, and J. J. Mortensen, J. Chem. Phys. 128, 244101 (2008).

${ }^{12}$ X. Zhang, Z. Li, and G. Lu, Phys. Rev. B 84, 235208 (2011).

${ }^{13}$ W. Mou, S. Ohmura, F. Shimojo, and A. Nakano, Appl. Phys. Lett. 100, 203306 (2012).

${ }^{14}$ T. Tsukagoshi and O. Sugino, Phys. Rev. A 86, 064501 (2012). 
${ }^{15}$ S. Baroni, S. De Gironcoli, A. D. Corso, and P. Giannozzi, Rev. Mod. Phys. 73, 515 (2001).

${ }^{16}$ A. Putrino, D. Sebastiani, and M. Parrinello, J. Chem. Phys. 113, 7102 (2000).

${ }^{17}$ J. Hutter, J. Chem. Phys. 118, 3928 (2003).

${ }^{18}$ T. L. Beck, Rev. Mod. Phys. 72, 1041 (2000).

${ }^{19}$ M. Heiskanen, T. Torsti, M. J. Puska, and R. M. Nieminen, Phys. Rev. B 63, 245106 (2001).

${ }^{20}$ D. Vanderbilt, Phys. Rev. B 41, 7892 (1990).

${ }^{21}$ P. E. Blochl, Phys. Rev. B 50, 17953 (1994).

${ }^{22}$ G. Kresse and D. Joubert, Phys. Rev. B 59, 1758 (1999).

${ }^{23}$ R. E. Stratmann, G. E. Scuseria, and M. J. Frisch, J. Chem. Phys. 109, 8218 (1998).

${ }^{24}$ C. van Caillei and R. D. Amos, Chem. Phys. Lett. 308, 249 (2000).

${ }^{25}$ F. Furche and R. Ahlrichs, J. Chem. Phys. 117, 7433 (2002).

${ }^{26}$ G. Kresse and J. Hafner, Phys. Rev. B 47, 558 (1993).

${ }^{27}$ G. Kresse and J. Furthmuller, Phys. Rev. B 54, 11169 (1996).

${ }^{28}$ J. P. Perdew and A. Zunger, Phys. Rev. B 23, 5048 (1981).

${ }^{29}$ D. J. Clouthier and D. A. Ramsey, Annu. Rev. Phys. Chem. 34, 31 (1983).

${ }^{30}$ M. J. Frisch, G. W. Trucks, H. B. Schlegel, G. E. Scuseria, M. A. Robb, J. R. Cheeseman, G. Scalmani, V. Barone, B. Mennucci, G. A. Petersson, H. Nakatsuji, M. Caricato, X. Li, H. P. Hratchian, A. F. Izmaylov, J. Bloino, G. Zheng, J. L. Sonnenberg, M. Hada, M. Ehara, K. Toyota, R. Fukuda, J. Hasegawa, M. Ishida, T. Nakajima, Y. Honda, O. Kitao, H. Nakai, T. Vreven, J. A. Montgomery, Jr., J. E. Peralta, F. Ogliaro, M. Bearpark, J. J. Heyd, E. Brothers, K. N. Kudin, V. N. Staroverov, T. Keith, R. Kobayashi, J. Normand,
K. Raghavachari, A. Rendell, J. C. Burant, S. S. Iyengar, J. Tomasi, M. Cossi, N. Rega, J. M. Millam, M. Klene, J. E. Knox, J. B. Cross, V. Bakken, C. Adamo, J. Jaramillo, R. Gomperts, R. E. Stratmann, O. Yazyev, A. J. Austin, R. Cammi, C. Pomelli, J. W. Ochterski, R. L. Martin, K. Morokuma, V. G. Zakrzewski, G. A. Voth, P. Salvador, J. J. Dannenberg, S. Dapprich, A. D. Daniels, O. Farkas, J. B. Foresman, J. V. Ortiz, J. Cioslowski, and D. J. Fox, GaUSSIAN 09, Revision B.01, Gaussian, Inc., Wallingford CT, 2010.

${ }^{31}$ T. Liu and A. Troisi, J. Phys. Chem. C 115, 2406 (2011).

${ }^{32}$ R. van Meer, O. V. Gritsenko, and E. J. Baerends, J. Chem. Theory Comput. 10, 4432 (2014).

${ }^{33}$ H. Iikura, T. Tsuneda, T. Yanai, and K. Hirao, J. Chem. Phys. 115, 3540 (2001).

${ }^{34}$ R. Baer and D. Neuhauser, Phys. Rev. Lett. 94, 043002 (2005).

${ }^{35}$ X. Zhang, Z. Li, and G. Lu, J. Phys.: Condens. Matter 24, 205801 (2012).

${ }^{36}$ F. Appel, E. K. U. Gross, and K. Burke, Phys. Rev. Lett. 90, 043005 (2003).

${ }^{37}$ R. Kobayashi and R. D. Amos, Chem. Phys. Lett. 420, 106 (2006).

${ }^{38}$ G. Sini, J. S. Sears, and J. L. Bredas, J. Chem. Theory Comput. 7, 602 (2011).

${ }^{39}$ T. Sato, T. Tsuneda, and K. Hirao, J. Chem. Phys. 123, 104307 (2005).

${ }^{40}$ E. Livshits and R. Baer, Phys. Chem. Chem. Phys. 9, 2932 (2007).

${ }^{41}$ J. C. Tully, J. Chem. Phys. 93, 1061 (1990).

${ }^{42}$ H. M. Jaeger, K. Hyeon-Deuk, and O. V. Prezhdo, Acc. Chem. Res. 46, 1280 (2013).

${ }^{43}$ S. Thomas, G. Joly, and T. Swager, Chem. Rev. 107, 1339 (2007).

${ }^{44}$ E. Tapavicza, I. Tavernelli, and U. Rothlisberger, Phys. Rev. Lett. 98, 023001 (2007).

${ }^{45}$ S. Hammes-Schiffer and J. C. Tully, J. Chem. Phys. 101, 4657 (1994). 